\title{
Uma investigação sobre o marketing de influência
}

\author{
Carlos Oliveira $^{1}$, Lívian Pascoal ${ }^{1}$, Luisa Veloso ${ }^{1}$, Victor Lima ${ }^{1}$, Letícia Viana ${ }^{1}$ \\ ${ }^{1}$ Instituto Federal do Rio de Janeiro (IFRJ) \\ carlos.robertodifrj.edu.br
}

\begin{abstract}
Resumo. Com bilhões de usuários em todo o mundo, as redes sociais têm sido cada vez mais utilizadas para a compra e venda de produtos e serviços. Alguns desses usuários, os influenciadores digitais, têm muitos "seguidores"nessas redes. Esses seguidores são usuários que acompanham as postagens dos influenciadores digitais. Algumas dessas postagens são divulgações de produtos e serviços.

Apesar de diversas empresas utilizarem as postagens dos influenciadores como estratégia de marketing, um relatório recente mostra que a taxa de conversão em vendas dessas postagens é baixa. Então, é necessário entender o que leva os usuários a comprar ou deixar de comprar produtos/serviços divulgados por esses influenciadores.

Nesta pesquisa utilizamos um questionário com 548 pessoas que fazem compras em redes sociais para entender quais fatores as fazem comprar ou deixar de comprar a partir das postagens dos influenciadores. Os resultados iniciais mostram que esses fatores estão relacionados as seguintes características do influenciador (1) credibilidade (ou falta dela), (2) ser especialista (ou não) no produto/serviço que está divulgando, (3) carisma (ou falta dela) e, (4) não passar a impressão para o seguidor que está postando apenas porque está sendo pago.

Estamos realizando algumas entrevistas para entender como os usuários identificam essas características a partir dos dados que estão disponíveis nas redes sociais. Por uma questão de espaço, a análise desses dados (dos questionários e entrevistas) será divulgado posteriormente. Esses dados nos permitirão implementar um processo para ajudar a decidir quais influenciadores digitais devem ser convidados para divulgar determinados produtos/serviços de forma a maximizar as vendas.
\end{abstract}

\section{Estado da arte}

Bilhões de pessoas em todo o mundo usam atualmente as redes sociais. Elas têm sido utilizadas para compartilhar e buscar informações, o que ajudou a impulsionar seu uso para a venda de produtos/serviços. Diferentes fatores levam os consumidores a comprar nas redes sociais, podemos citar seis fatores: confiança [Li 2019], preço [Babutsidze 2018], qualidade do produto [Chen and Lin 2019], endosso de influenciador digital [Casaló et al. 2018], divulgação boca-a-boca [Zhang et al. 2020], e publicidade [Shang et al. 2017].

Esta pesquisa pretende focar especificamente no estudo do endosso de influenciadores. A característica das interações sociais permitiu que alguns usuários se destacassem e se tornassem influenciadores. Os influenciadores são pessoas que dominam o 
uso das redes sociais e que reuniram vários seguidores que "ouvem"o que eles postam [Chatterjee 2011].

Casaló et al. [Casaló et al. 2018] observaram que quando o consumidor sente que tem uma relação próxima com o influenciador isso influencia sua intenção de comprar o que é divulgado pelo influenciador. Esses consumidores tendem a aceitar o endosso do influenciador e a comprar o produto anunciado, mesmo que não haja identificação do influenciador com a marca [Carlson et al. 2020]. A interação parassocial (a forma como os usuários percebem sua relação com o influenciador [Gong and Li 2017]), e o capital social também afetam a intenção de compra do usuário [Kim et al. 2018]. A mídia social favorece interações parassociais entre seguidores e influenciadores [Lee and Watkins 2016]. Por meio da interação parassocial, os influenciadores fazem seus seguidores sentirem que são seus amigos [Gong and Li 2017] e é afetado positivamente pela credibilidade dos influenciadores [Yuan et al. 2016].

Chinchanachokchai e de Gregorio [Chinchanachokchai and de Gregorio 2020] descobriram que os consumidores confiam mais nas informações transmitidas por outros consumidores do que nos profissionais de marketing para tomar suas decisões de compra. Portanto, uma boa estratégia poderia ser apresentar anúncios por influenciadores digitais em vez das próprias empresas. No entanto, os seguidores valorizam as motivações intrínsecas e a orientação não comercial dos influenciadores. Assim, as postagens pagas podem resultar em dificuldades para a autenticidade dos influenciadores [Audrezet et al. 2018]. Com base em observações de postagens pagas e entrevistas com influenciadores, Audrezet, Kerviler, e Moulard [Audrezet et al. 2018] descobriram que uma maneira de o influenciador manter sua autenticidade é deixar claro aos seguidores que ele está sendo pago pela postagem.

\section{Questões de pesquisa}

Os influenciadores digitais fazem parte de uma estratégia maior que inclui campanhas de mídia social. Chatterjee [Chatterjee 2011] afirma que para novos produtos em categorias de produtos amplamente adquiridos, gerar um comentário ou apenas saber da existência de um novo produto pode ser suficiente para acionar a transmissão rápida de mensagens por meio da interação consumidor-consumidor, garantindo o sucesso. No entanto, para produtos com atributos de credibilidade ou aqueles que apresentam riscos significativos (como produtos para a saúde), a consciência por si só pode não ser suficiente; a capacidade do influenciador de fornecer informações que mitigam o risco percebido e induzem seus seguidores a se envolver com a marca é fundamental para o sucesso na estratégia de mídia social.

Porém, as empresas precisam ser extremamente cuidadosas, pois o valor pago ao influenciador não garante bons resultados. Uma má escolha de influenciadores para uma campanha pode levar a um desperdício de dinheiro e também à desconfiança do consumidor em relação a essa campanha ${ }^{1}$. De acordo com um recente relatório ${ }^{2}$, as taxas de conversão para vendas online nas principais plataformas de mídia social, como Twitter e Snapchat, foram surpreendentemente baixas, com menos de 1\%. Dada a popularidade

\footnotetext{
${ }^{1}$ https://zap.aeiou.pt/empresas-mil-milhoes-euros-influencers-272982

${ }^{2}$ https://www.forbes.com/sites/priceonomics/2018/03/09/the-advertising-conversion-rates-for-everymajor-tech-platform/65454d435957
} 
do engajamento de comércio de mídia social versus o fracasso das empresas em atingir o desempenho esperado, cada vez mais atenção acadêmica tem sido dada a essa área de pesquisa.

Embora vários estudos tenham pesquisado o endosso dos influenciadores nas redes sociais, os pesquisadores se concentraram em questões específicas, por exemplo:

1. Lee e Watkins [Lee and Watkins 2016] estudaram como as percepções dos consumidores sobre marcas de luxo são afetadas por vídeos de Youtubers;

2. Djafarova e Rushworth [Djafarova and Rushworth 2017] investigaram o impacto do Instagram na credibilidade do influenciador e na intenção de compra do usuário, além da identificação do seguidor com o influenciador;

3. Albert, Ambroise, e Valette-Florence [Albert et al. 2017] investigaram como a identificação do consumidor com a marca influencia sua intenção de compra;

4. Fink et al. [Fink et al. 2018] estudaram como o uso de influenciadores pode impulsionar as intenções de compra de longo prazo (quatro anos em seu trabalho).

Porém, é preciso entender quais fatores são decisivos para que o seguidor (quem vai consumir o que é divulgado) efetue a compra. Também é necessário identificar como mapear esses fatores em dados que podem ser coletados nas redes sociais. Portanto, queremos identificar (por meio de questionários e entrevistas com seguidores/consumidores nas redes sociais) o que leva o seguidor a comprar um produto/serviço do divulgado pelo influenciador. Portanto, temos as seguintes questões de pesquisa:

1. Quais características do influenciador levam o seguidor a comprar um produto/serviço?

2. Quais características do influenciador levam o seguidor a deixar de comprar um produto/serviço?

\section{Proposta de solução}

Este projeto pretende criar um processo que possa ser utilizado para definir os influenciadores digitais mais adequados para divulgar um determinado produto/serviço. Esse processo será baseado nas variáveis que influenciam a decisão de compra dos usuários das redes sociais (que serão identificadas por meio de revisão de literatura, entrevistas e questionários). Este processo permitirá que sejam criados sistemas de informação para identificar os influenciadores mais adequados (a partir de um conjunto disponível) para divulgar um determinado produto/serviço.

\section{Metodologia de pesquisa}

Até o momento, foi realizada e publicada uma revisão sistemática da literatura, como primeiro artigo deste projeto. Os resultados desta revisão sistemática mostraram que a interação parassocial (a forma como os usuários percebem sua relação com o influenciador) [Gong and Li 2017], e o capital social afeta a intenção de compra do usuário [Kim et al. 2018]. A mídia social favorece interações parassociais entre seguidores e influenciadores [Lee and Watkins 2016]. Por meio da interação parassocial, os influenciadores fazem seus seguidores sentirem que são seus amigos [Gong and Li 2017] e são afetados positivamente pela credibilidade dos influenciadores [Yuan et al. 2016]. 
Após a revisão sistemática, conduzimos um questionário (Apêndice 1) com 548 usuários que utilizam as redes sociais para comprar ou buscar informações antes de realizar suas compras. Um dos objetivos do questionário era verificar se os fatores identificados na revisão de literatura afetam as intenções de compra dos usuários e se os respondentes iriam apresentar outros fatores.

Neste momento, estamos realizando entrevistas semi-estruturadas (as perguntas são apresentadas no (Apêndice 2) com alguns respondentes do questionário para entender como esses usuários identificam esses fatores a partir dos dados disponíveis nas redes sociais. As entrevistas serão realizadas e codificadas até atingirmos a saturação teórica [Muller 2014]. Nosso objetivo é desenvolver um código para implementar o modelo de escolha dos influenciadores que devem fazer uma divulgação. Este código irá coletar as variáveis que forem identificadas nas entrevistas.

A análise dos dados do questionário será feita utilizando testes estatísticos. A análise dos dados das entrevistas será feita utilizando o sistema de codificação apresentado na Teoria Fundamentada em Dados [Glaser and Strauss 1967], que é realizada em três passos [Strauss and Corbin 1990]: codificação aberta, codificação axial e codificação seletiva.

A codificação aberta pode ser conceituada como o processo analítico pelos quais os conceitos são identificados e desenvolvidos em relação às suas propriedades e dimensões. Esse processo envolve as atividades de quebrar, examinar, comparar, conceituar e categorizar os dados que serão sumarizados em uma lista de códigos e categorias originadas dos rótulos atribuídos livremente a cada frase, linha ou parágrafo [Corbin 1990]. Para rotular os dados, utilizam-se perguntas e comparações em busca de similaridade e diferenças entre cada incidente, evento ou situação. Portanto, utilizam-se perguntas como "o que isso representa?"[Corbin 1990]. Os eventos e incidentes semelhantes são comparados e agrupados para formar categorias.

O objetivo na etapa da codificação axial consiste em aprimorar e diferenciar as categorias resultantes da codificação aberta. Nessa etapa são selecionadas as categorias mais relevantes e são colocadas como fenômeno central para estabelecer as relações entre as categorias e subcategorias. Portanto, nesta etapa os dados são colocados em uma nova forma, por meio das relações entre as categorias. Isto é realizado com o paradigma de codificação que envolve condições, contexto, estratégias de ação/interação e suas consequências [Corbin 1990].

A codificação seletiva é a terceira e última etapa de codificação. Nesta etapa, o objetivo é integrar e refinar categorias em um nível mais abstrato. A tarefa é elaborar a categoria essencial, em torno da qual as outras categorias desenvolvidas possam ser agrupadas e pelas quais são integradas. Segundo Strauss e Corbin [Corbin 1990], ”o fenômeno central é o coração do processo de integração". Portanto, tanto na codificação aberta quanto na axial, os fenômenos foram nomeados para que na codificação seletiva o pesquisador possa olhar a lista de categorias e avaliar qual delas é abstrata o suficiente para englobar todas as outras que foram descritas.

Teorias comportamentais também serão usadas para ajudar a entender o comportamento do comprador (seguidor do influenciador) nas redes sociais. A Teoria da Influência Social [Kelman 1958] será utilizada para mostrar o processo de aceitação da influência 
(influência aqui significa que o usuário/possível comprador fará algo que não faria na ausência da influência, ou seja, comprar a partir da postagem). Também serão utilizadas teorias para explicar se a compra (do seguidor/comprador) a partir da postagem do influenciador é um comportamento planejado [Ajzen et al. 1991] ou não [Norman 2013].

Entender o comportamento do comprador é importante porque nos permitirá propor um processo, que pretendemos implementar. Este processo ajudará a escolher os influenciadores que devem ser convidados a divulgar um determinado produto/serviço para maximizar as vendas.

\section{Resultados parciais e trabalhos futuros}

A partir dos questionários que conduzimos com 548 usuários que fazem compras nas redes sociais, observamos quatro características que os levam a comprar ou deixar de comprar nas redes sociais. São elas, (1) credibilidade (ou falta dela), (2) ser especialista (ou não) no produto/serviço que está divulgando, (3) carisma (ou falta dela) e, (4) não passar a impressão para o seguidor que está postando apenas porque está sendo pago.

$\mathrm{Na}$ etapa atual deste trabalho, estamos realizando entrevistas para entender como os usuários identificam essas características a partir dos dados que estão disponíveis nas redes sociais. Esse entendimento nos permitirá saber quais dados, daqueles disponíveis nas redes sociais, devem ser utilizados para implementar um processo para a escolha dos influenciadores que devem divulgar determinados produtos/serviços de forma a maximizar as vendas.

Atualmente também estamos trabalhando na coleta de dados do Twitter para implementar o processo que pretendemos propor. Com o uso do Facepager 4.3 extraímos dados do Twitter usando a API 1.1, que nos permite automatizar o processo de coleta de dados da rede social. O dados coletados são lidos pelos aplicativo Gephi e apresentados na forma de gráficos. No estágio atual do trabalho, estamos treinando a extração e apresentação dos dados. No momento em que esta versão do texto é submetida, não foi feita ainda a análise dos dados das entrevistas. Portanto, ainda não definimos quais variáveis devem ser coletadas. Após a análise dos dados coletados nas entrevistas, poderemos propor, implementar e avaliar um processo para a escolha dos influenciadores que devem ser convidados para divulgar produtos e serviços.

\section{Referências}

Ajzen, I. et al. (1991). The theory of planned behavior. Organizational behavior and human decision processes, 50(2):179-211.

Albert, N., Ambroise, L., and Valette-Florence, P. (2017). Consumer, brand, celebrity: Which congruency produces effective celebrity endorsements? Journal of Business Research, 81:96-106.

Audrezet, A., De Kerviler, G., and Moulard, J. G. (2018). Authenticity under threat: When social media influencers need to go beyond self-presentation. Journal of Business Research.

Babutsidze, Z. (2018). The rise of electronic social networks and implications for advertisers. Technological Forecasting and Social Change, 137:27-39. 
Carlson, B. D., Donavan, D. T., Deitz, G. D., Bauer, B. C., and Lala, V. (2020). A customer-focused approach to improve celebrity endorser effectiveness. Journal of Business Research, 109:221-235.

Casaló, L. V., Flavián, C., and Ibáñez-Sánchez, S. (2018). Influencers on instagram: Antecedents and consequences of opinion leadership. Journal of Business Research.

Chatterjee, P. (2011). Drivers of new product recommending and referral behaviour on social network sites. International Journal of Advertising, 30(1):77-101.

Chen, S.-C. and Lin, C.-P. (2019). Understanding the effect of social media marketing activities: The mediation of social identification, perceived value, and satisfaction. Technological Forecasting and Social Change, 140:22-32.

Chinchanachokchai, S. and de Gregorio, F. (2020). A consumer socialization approach to understanding advertising avoidance on social media. Journal of Business Research, 110:474-483.

Corbin, J. M. (1990). Basics of qualitative research: Grounded theory procedures and techniques. Sage.

Djafarova, E. and Rushworth, C. (2017). Exploring the credibility of online celebrities' instagram profiles in influencing the purchase decisions of young female users. Computers in Human Behavior, 68:1-7.

Fink, M., Koller, M., Gartner, J., Floh, A., and Harms, R. (2018). Effective entrepreneurial marketing on facebook-a longitudinal study. Journal of business research.

Glaser, B. and Strauss, A. (1967). Discovery of grounded theory. strategies for qualitative research sociology press.".

Gong, W. and Li, X. (2017). Engaging fans on microblog: The synthetic influence of parasocial interaction and source characteristics on celebrity endorsement. Psychology \& Marketing, 34(7):720-732.

Kelman, H. C. (1958). Compliance, identification, and internalization three processes of attitude change. Journal of conflict resolution, 2(1):51-60.

Kim, J., Kang, S., and Lee, K. H. (2018). How social capital impacts the purchase intention of sustainable fashion products. Journal of Business Research.

Lee, J. E. and Watkins, B. (2016). Youtube vloggers' influence on consumer luxury brand perceptions and intentions. Journal of Business Research, 69(12):5753-5760.

Li, C.-Y. (2019). How social commerce constructs influence customers' social shopping intention? an empirical study of a social commerce website. Technological Forecasting and Social Change, 144:282-294.

Muller, M. (2014). Curiosity, creativity, and surprise as analytic tools: Grounded theory method. In Ways of Knowing in HCI, pages 25-48. Springer.

Norman, D. (2013). The design of everyday things: Revised and expanded edition. Basic books.

Shang, S. S., Wu, Y.-L., and Sie, Y.-J. (2017). Generating consumer resonance for purchase intention on social network sites. Computers in Human Behavior, 69:18-28. 
Strauss, A. and Corbin, J. (1990). Basics of qualitative research. Sage publications.

Yuan, C. L., Kim, J., and Kim, S. J. (2016). Parasocial relationship effects on customer equity in the social media context. Journal of Business Research, 69(9):3795-3803.

Zhang, H., Liang, X., and Qi, C. (2020). Investigating the impact of interpersonal closeness and social status on electronic word-of-mouth effectiveness. Journal of Business Research.

\section{Apêndice}

\subsection{Apêndice 1: Perguntas do questionário}

Q1: Que tipo de produtos você costuma comprar nas redes sociais (ou após ver a postagem de um influenciador digital)?

1. Eletrônicos

2. Produtos / serviços de beleza

3. Jogos

4. Livros

5. Serviços/produtos relacionados a viagens

6. Itens para casa

7. Alimentos

8. Vestuário

9. Viagens

10. Outros

Q2: Em relação a compras a partir de postagens em redes sociais, escolha as opções abaixo que refletem a sua realidade.

1. Faço (ou já fiz) compras após ver a postagem de um influenciador digital, mesmo que inicialmente eu não tivesse a intenção de comprar o que estava sendo anunciado

2. Quando tenho a intenção de fazer uma compra, utilizo a rede social para buscar informações sobre o produto/serviço antes de realizar a compra

3. Já deixei de fazer uma compra (que tinha a intenção de fazer) após ver uma postagem na rede social sobre o produto

4. Não faço compras influenciado(a) pelas redes sociais

Q3: Caso você busque informações nas redes sociais antes de fazer uma compra, em qual tipo de perfil você busca essas informações?

1. Influenciadores digitais com muitos seguidores

2. Influenciadores digitais especialistas no assunto, não necessariamente que tenham muitos seguidores

3. Meus amigos

4. Empresas

5. Qualquer pessoa que fale sobre o produto/serviço que estou buscando

6. Outro

Q4: Quais dos fatores abaixo seriam necessários para que você faça uma compra a partir da postagem de um influenciador?

1. Credibilidade 
2. Ser especialista no produto/serviço

3. Carisma

4. Outro

Q5: Quais dos fatores abaixo podem te levar a DEIXAR de fazer uma compra a partir da postagem de um influenciador?

1. Falta de credibilidade

2. Perceber que ele não entende do produto/serviço que está divulgando

3. Eu não me identificar com o influenciador (ter opiniões diferentes)

4. Achar que ele só está falando daquele produto/serviço porque está sendo pago

5. Incoerência com o nicho do qual ele é

6. Outro

\subsection{Apêndice 2: Perguntas das entrevistas semi-estruturadas}

Q1: Qual a sua idade?

Q2: Qual a sua profissão?

Q3: Quais redes sociais você utiliza?

Q4: Aproximadamente, quantas pessoas você segue nas redes sociais?

Q5: Você segue as mesmas pessoas/perfis em todas as redes sociais? (A ideia aqui é ver se a pessoa usa uma rede pra lazer, outra pra trabalho, possivelmente concentra as compras em alguma..)

Q6: Você compra, ou já comprou, algum produto anunciado nas redes sociais?

Q7: Que tipo de produto você compra a partir desses anúncios?

Q8: Geralmente, esses anúncios que te levaram a fazer uma compra foram feitos por qual tipo de perfil? (influenciador/amigo/qualquer um..)

Q9: Além das compras depois de ver um anúncio, você costuma utilizar as redes sociais para buscar informações antes de fazer uma compra?

Q10: Qual (ou quais) rede(s) você utiliza para buscar essas informações? rede social

Q11: Com relação aos influenciadores digitais, a partir do que está disponível na

1. a) O que você entende como credibilidade do influenciador?

2. b) Como você avalia se ele tem credibilidade?

3. c) Como você avalia se ele NÃO tem credibilidade?

4. d) Quando ele divulga algum produto ou serviço, como você avalia se ele é especialista naquele produto ou serviço?

5. e) $\mathrm{O}$ que pode te levar a pensar que ele não entende do produto/serviço que está divulgando?

6. f) O que pode te levar a pensar que ele está falando daquele produto/serviço apenas porque está sendo pago?

7. g) O que pode te levar a pensar que o que ele está divulgando é incoerente com o nicho de postagens dele?

8. h) Como você avalia se um influenciador tem carisma ou não?

9. i) Como você avalia que você não se identifica com o influenciador? 